\title{
Epidermal cysts - a clinicopathological and biochemical study
}

\author{
V. ChandRasekaran* \\ M.S.
}

\author{
SATYA PARKaSH* \\ F.R.C.S. (Eng.)
}

\author{
C. V. Raghuveer $\dagger$ \\ M.D., D.C.P. \\ Departments of * Surgery and $\dagger$ Pathology, \\ Jawaharlal Institute of Postgraduate Medical Education and Research, \\ Pondicherry 605006, India
}

\begin{abstract}
Summary
The findings of a clinicopathological and biochemical study of epidermal cysts and a review of the relevant literature are presented. A punctum was found in $\mathbf{4 0} \%$ of 34 epidermal cysts that were studied in detail. The findings of the histological study were in favour of the punctum being the orifice of an obstructed hair follicle from which at least a proportion of the epidermal cysts are likely to develop. The biochemical analysis of the contents of the cysts revealed a very low protein and lipid content, thus ruling out any 'sebaceous' contribution. Bacteriological study of clinically inflamed cysts showed that inflammation in these lesions was usually aseptic unless there was a communication between the cyst cavity and the exterior.
\end{abstract}

\section{Introduction}

Sebaceous cyst is the most common diagnosis offered whenever a cystic lesion of the skin is encountered in surgical practice. As the contents of these lesions appear to be sebaceous on casual examination, it was for long presumed that these lesions resulted from blockage of the sebaceous ducts with the consequent retention of the sebaceous secretion. Although work published by pathologists and dermatologists has disproved this theory beyond all doubt, surgical text books, by and large, continue to use this term and subscribe to this discredited theory. As a result, much confusion and misconception exist among surgeons regarding the true nature of these lesions. This study was undertaken, therefore, in an attempt to study these common cystic lesions by correlating their clinical characteristics with the underlying pathology and the biochemical nature of their contents.

Although it is not clear as to when exactly the term 'sebaceous cyst' came into use, the 'sebaceous concept' could be traced to the great pathologist
Rudolf Virchow, who considered all epitheliumlined cysts of the skin with semi-solid or fluid contents to be formed from sebaceous glands (Warvi and Gates, 1943). The term 'sebaceous cyst' has been used to describe most, if not all, cystic lesions of the skin. However, as far back as 1930, Broders and Wilson classified these lesions on the basis of histology and physical characteristics of their contents into 'keratomas' and true sebaceous cysts. In 1943, Warvi and Gates and Love and Montgomery made separate studies and arrived at similar conclusions although they preferred to call the 'keratomas' of Broders and Wilson as 'epidermal cysts' and 'epithelial cysts' respectively. Kligman in 1964 studied 13 of the so-called sebaceous cysts and concluded that they were nothing more than a variety of keratinous cyst. McGavran and Binnington (1966) made a detailed study of all the characteristics of 'sebaceous cysts' including their histochemical reactivity and mode of keratinization, as observed under the electron microscope. They concluded that the keratinous cysts, wherein keratinization occurred without keratohyaline granules, as in the cases of the cortex of hair and nail, were derived from the piliary apparatus, particularly the external root sheath and hence should be called 'pilar cysts'. In contrast to this, they found that keratinization in the more generally distributed epidermal cysts occurred with the formation of keratohyaline granules as in the case of epidermis. Nicoloides, Leven and $\mathrm{Fu}$ (1968) analysed the lipid pattern of epidermal and the so-called sebaceous cysts and found that neither of them resembled the lipid pattern of the sebaceous gland secretions. Pinkus (1969), while accepting the pilar theory, identified the follicular isthmus of the external root sheath of the hair follicle as the exact origin of these pilar cysts and suggested the name 'trichilemmal cysts'. 
Thus, at present it is fairly clear that, except for steatocystoma multiplex, a rare and inherited disorder, there are 2 main types of skin cyst, the epidermal and the pilar, in place of the non-existent 'sebaceous cyst'. While the origin of pilar cyst is now well understood, the histogenesis of the epidermal cyst is still not clear. Various theories have been postulated from time to time. Sebaceous ducts (Broders and Wilson, 1930), rudimentary cell rests (Love and Montgomery, 1943), pluripotent epithelial systems of epidermis (Kligman, 1964) and occluded pilosebaceous follicle (Aurora and Blodi, 1972; Caro, 1975), have all been suggested as the structures of origin. Implantation dermoids, which are a variety of epidermal cyst, are generally considered to be caused by trauma, either blunt or penetrating (Epstein and Kligman, 1957; Sanderson and Machie, 1979).

\section{Materials and methods}

A clinico-pathological study of all the cases of epidermal and pilar cysts subjected to histology during the period January 1978 to September 1979 was made from the Institute Hospital records. Of these, 34 consecutive epidermal cysts and one pilar cyst were subjected to a critical study which included a detailed clinical examination, a histological study and a biochemical analysis of the contents for total proteins and lipids. During the clinical examination, a special effort was made to find out the presence of a punctum, using a magnifying glass $(\times 10)$ whereever necessary. In the case of cysts having a punctum, 3 to 5 serial sections at a distance of $3 \mu$ were made through the punctum. The sections were stained with haematoxylin and eosin and studied under the microscope for the structure of the cyst wall, the appearance of the contents and the exact nature of the punctum. Estimation of total proteins (Biuret method) and lipids (Gravimetric method) in the undehydrated cyst contents was done in 15 epidermal cysts including 4 implantation dermoids. Five subcutaneous dermoids and 3 teratomatous dermoids containing a dermal element were also subjected to a similar biochemical study for comparison. Only those cysts which had no clinical signs of inflammation and were removed with an intact cyst wall were accepted for biochemical analysis to exclude contamination with tissue fluids and blood. The content of the only pilar cyst studied could not be biochemically analysed as it had burst and was inflamed at the time of examination. The contents of 4 inflamed epidermal cysts, one implanatation dermoid and on pilar cyst were also subjected to a bacteriological culture.

\section{Results}

During the study, 249 epidermal or 'sebaceous' cysts were diagnosed at the surgical outpatient department of the Jipmer Hospital, of which only 123 cysts $(41.8 \%)$ were subjected to histological examination. Out of these 123,2 were pilar $(1.58 \%)$ and the rest epidermal cysts.

The 121 histologically proved epidermal cysts were from 84 male and 37 female patients, a male to female ratio of $2: 1$. The oldest patient was 70 years old and the youngest 3 years (average, 29.3 years). The highest incidence occurred in the 3rd and 4th decades. There was a wide variation in duration from 3 months to 30 years (average, $3 \cdot 2$ years). The commonest site was the face $(19.0 \%)$ followed by the chest wall $(12.41 \%)$, thigh $(10.74 \%)$, gluteal region $(8.34 \%)$ and scalp $(8.26 \%)$. The largest cyst (gluteal region) was $9 \mathrm{~cm}$ and the smallest (scrotum) was $0.5 \mathrm{~cm}$. The lesions were mostly single except for scrotal cysts which were multiple lesions in all 5 cases.

Of the 34 consecutive cysts studied in detail, 15

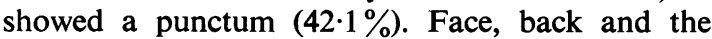
gluteal region were the most frequent sites. In all 15 cases, the punctum was visible to the naked eye. Clinically it appeared as a small dark opening through which the cyst contents could be squeezed out, or as a minute pit, where the skin was adherent to the

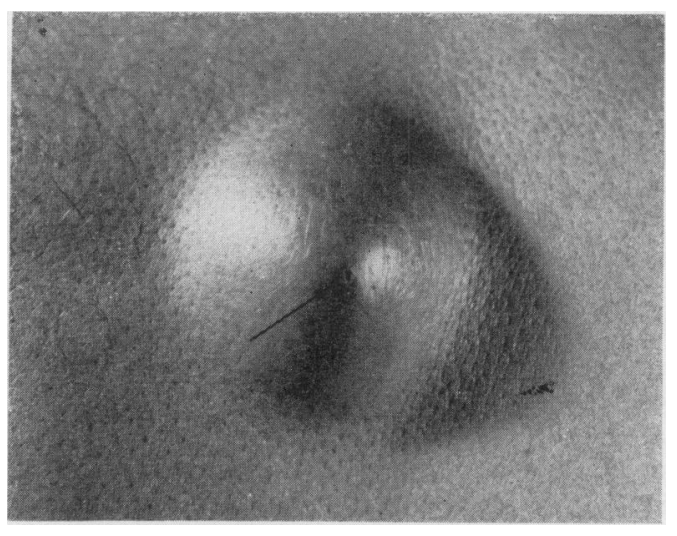

FIG. 1. Epidermal cyst with purctum (arrowed)

underlying cyst (Figs 1 and 2). Six of 34 cysts $(17.6 \%)$ had histological evidence of inflammation. Of these, only 5 were clinically inflamed. Two cases of multiple cysts of the scrotum had clinical evidence of calcification; and one cyst occurring in the cheek had microscopical evidence of calcification, giving an overall incidence of calcification of $8.8 \%$.

Both cases of pilar cyst occurred on the scalp of women. Of these, only one, in a 50-year-old woman, was studied in detail: it was $3 \mathrm{~cm}$ in size and was of 
30 years' duration. The patient had, while combing her hair, accidentally traumatized the cyst one week before examination. At the time of examination the cyst was collapsed, tender and was discharging scanty, thick yellowish fluid through a ragged opening near its base.

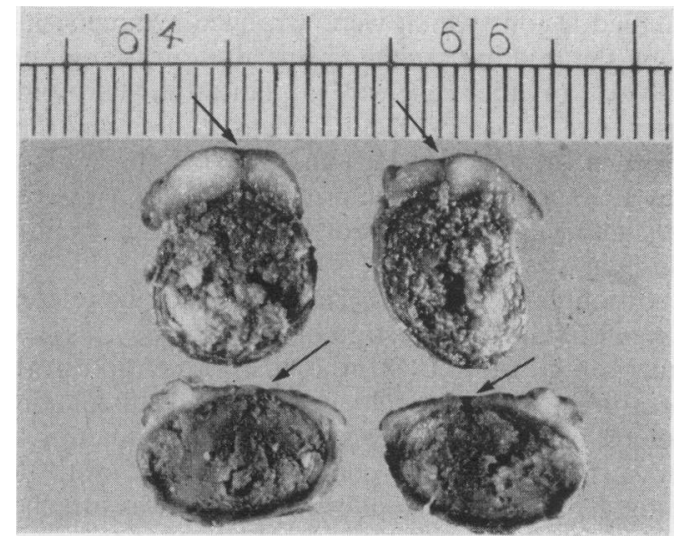

Fig. 2. Punctum (arrowed) as seen in the cut section of two epidermal cysts.

Of 121 cases of epidermal cysts 10 were cysts occurring in the recognized site of implantation dermoids namely the palms, fingers and soles and could be classified as implantation dermoids - an incidence of $8.3 \%$ ( 8 male and 2 female patients): all but one of the men were manual labourers; none gave a history of antecedent trauma. While 6 cysts occurred in the fingers, one each occurred in the palm and the inter-digital cleft and 2 in the soles. Significantly only one of the 6 cysts seen in the fingers was situated in the finger tip, the site most commonly exposed to trauma. Similarly both the cysts occurring in the soles were also situated in non-weight-bearing areas, namely the medial aspect of the ball of the great toe and the web space. Two of the implantation dermoids had evidence of inflammation.

On histological examination, all the epidermal cysts including the implantation dermoids were found to have an epidermal lining with a distinct granular cell layer and laminated keratin material forming the content. The lining was of variable thickness and the cells appeared to become flattened as they moved towards the cyst cavity (Fig. 3). One of the cysts had a portion of disintegrated hair shaft in the cyst cavity. In contrast to this, the pilar cysts showed a lining composed of several layers of pale epithelial cells with indistinct cell outlines. The cells appeared to become swollen as they moved towards

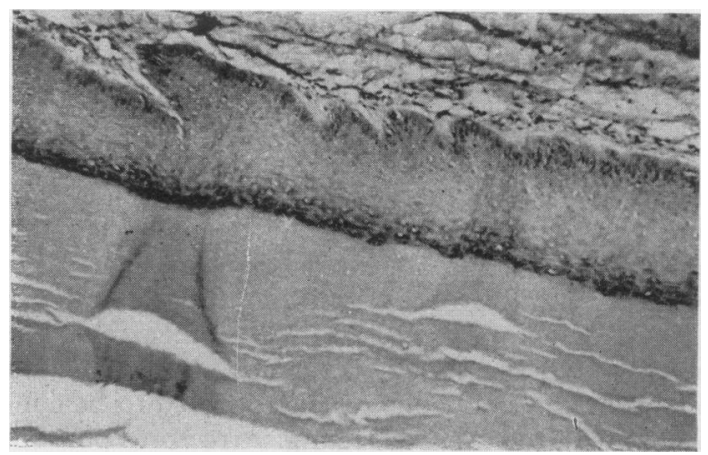

FIG. 3. Epidermal cyst showing typical epidermal lining and laminated keratin $(\mathrm{HE}, \times 100)$,

the cavity, changing abruptly into an amorphous keratin without the formation of a granular cell layer (Fig. 4). In the case of inflamed cysts, there were one or more breaches in the cyst wall where the epidermal lining was replaced by inflammatory cells. Extensive calcification of the cyst cavity could be

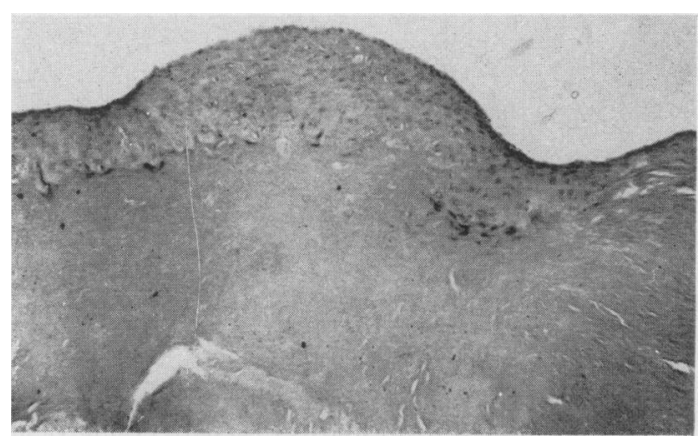

FIG. 4. Pilar cyst showing typical lining $(\mathrm{HE}, \times 100)$.

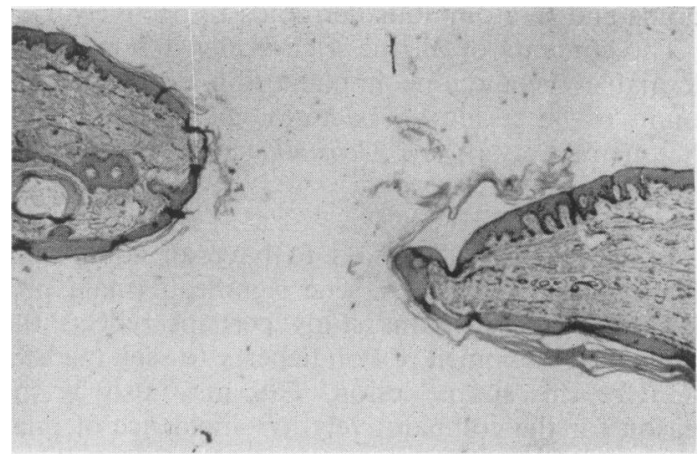

FIG. 5. Punctum seen as an opening, through which the cyst lining is continuous with the surface epidermis $(\mathrm{HE}, \times 100)$. 
seen in the 2 cases of multiple epidermal cyst of the scrotum studied in detail. The epidermal lining was markedly attenuated and barely discernible at places.

Of the 15 cysts subjected to serial sections, the punctum could be demonstrated in 4 and less distinctly in one. In 3 of these the punctum appeared to be a minute opening through which the epidermal lining of the cyst wall appeared to be continuous with the surface epidermis (Fig. 5). In the other cases the punctum was represented by an area of invagination of the surface epidermis which appeared to be connected with the cyst wall lining. Arrectores pilorum muscle could be seen attached to this connection in at least one case (Fig. 6).

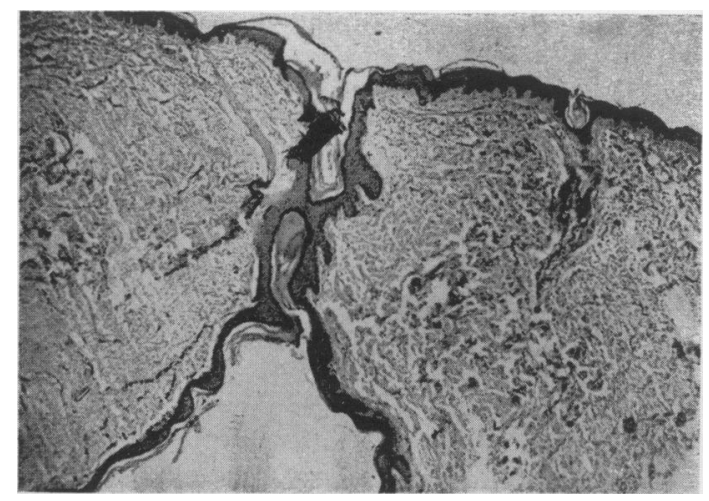

FIG. 6. Punctum showing the connection between the surface epidermis and the cyst lining and the arrectores pilorum muscle.

The biochemical examination revealed $0 \cdot 49,0 \cdot 54$, 0.88 and $0.85 \mathrm{~g} \%$ of protein; and $0.97,0.74,0.96$, and $1.28 \mathrm{~g} \mathrm{\%}$ of lipids in the content of epidermal cysts, implantation dermoids, sequestration dermoids and teratomatous dermoids respectively.

The contents of all the 4 clinically inflamed epidermal cysts and one implantation dermoid were found to be sterile on bacteriological culture, but the one pilar cyst grew Klebsiella sp.

\section{Discussion}

Epidermal cysts are said to have an equal incidence among the sexes. The significant male predominance seen in this study perhaps reflects the reluctance of women of Pondicherry to seek medical help for this simple lesion. This may also be the reason for the comparatively low incidence of pilar cysts which are known to occur predominantly in women (Caro, 1975). Contrary to the observations of Leppard, Sanderson and Wells (1977a) and
Sanderson and Machie (1979), there was no evidence of an autosomal dominant type of inheritance in the 2 cases studied.

There were certain special clinical features in the 5 cases of epidermal cyst of the scrotum in the present study which, so far as the authors know, have not been stressed in the literature: all 5 cases were of very long duration (10-30 years) and had multiple calcified lesions which were arranged symmetrically along the midline raphae in distribution though not is in size. In the case of implantation dermoids, $\vec{\circ}$ absence of a history of antecedent trauma and their presence in areas least exposed to trauma indicate that the theory of traumatic origin is not entirely satisfactory although trauma may be an exciting factor in a few cases.

Although it has long been known that a punctum is seen only in a proportion of epidermal cysts (Love and Montgomery, 1943), there is no mention in the literature of its exact incidence. As for its histological picture, in 2 cases it was seen as an invagination of the surface epidermis connected to the cyst wall lining. That arrectores pilorum muscle was attached to this connection is strong evidence that the punctum here actually represents the orifice of the obstructed hair follicle. It is possible that this represents an earlier stage in the development of a punc tum. The other 3 cases where the punctum was seep as an opening connecting the cyst cavity with the exterior probably represent a later stage, where the increasing pressure in the cyst cavity may have 'reopened' the obstructed pilosebaceous canal. What causes the initial obstruction of hair follicles which eventually distends to form a cyst, is a matter for speculation. Keratotic plugging (Auroroa and Blodi, 1972) seems a reasonable explanation in some of these cases. That one of the cysts showed a piece of disintegrated hair shaft in the cyst cavity appears to corroborate this view. However, it may also be argued that the gross stretching of a vicinity hair follicle may, in some cases, lead to follicle atrophy and a patulous opening overlying the cyst which resembles a punctum.

The very low lipid and protein contents indicate that there is no sebaceous contribution to the cyst content which is essentially composed of epithelial debris and keratin and is no different from similar material occurring elsewhere such as the subpreputial collection (Parkash et al., 1973). That bacteria could be isolated only from the one ruptured pilar cyst accords with the view of Leppard, Thomson and Noble (1977b) that inflammation in these cysts is usually an aseptic one caused by the cyst contents coming into contact with the surrounding tissues unless there is a communication with the exterior, when bacterial contamination can occur. 


\section{References}

Aurora, A. L. \& Blodi, F. C. (1972) Benign epithelial cysts of the eyelids. In: Current Concepts in Ophthalmology, Vol. III (Ed by Blodi, F. C.), p. 206. The C. V. Mosby Company, Saint Louis.

Broders, A. C. \& Wilson, R. (1930) Keratoma - a lesion often mistaken for sebaceous cyst. Surgical Clinics of Noth America, 10, 127.

Caro, W. A. (1975) Epithelial cysts. In: Dermatology Vol. 2 (Ed by Moschella, S. L., Pillsbury, D. M. \& Hurley, H. J.), 2nd Edn, p. 1329. Saunders Company, Philadelphia, London and Toronto.

Epstein, W. L. \& Kligman, A. N. (1957) Epithelial cysts in buried human skin. Archives of Dermatology, 76, 437.

Kligman, A. N. (1964) The myth of the sebaceous cyst. Archives of Dermatology, 89, 253.

Leppard, B. J., Sanderson, K. V. \& Well.s, R. S. (1977a) Hereditary tricholemmal cysts. Clinical and Experimental Dermatology, 2, 25.

Leppard, B. J., Thomson, J. P. S. \& Noble, W. C. (1977b)
Bacteriology of skin cysts. British Journal of Dermatology, 96, 511.

Love, W. R. \& Montgomery, H. (1943) Epithelial cysts. Archives of Dermatology and Syphilology, 47, 185.

McGavran, M. H. \& Binnington, B. (1966) Keratinous cysts of skin. Archives of Dermatology, 94, 499.

Nicoloides, N., Leven, N. E. \& Fu, H. C. (1968) The lipid pattern of the wen. Journal of Investigative Dermatology, 50, 189.

Parkash, S., Jeyakumar, S., Subramanyan, S. \& Chaudhri, S. (1973) Human subpreputial collection - its nature and formation. Journal of Urology, 110, 211.

Pinkus, H. (1969) 'Sebaceous cysts' are trichilemmal cysts. Archives of Dermatology, 99, 544.

Sanderson, K. V. \& Machie, R. (1979) Cysts of the skin. In: Text Book of Dermatology (Ed by Rook, A., Wilkinson, D. S. \& Ebling, F. J. G.), 3rd edn, Vol. 2, p. 2158. Blackwell Scientific Publications, Oxford, London, Edinburgh and Melbourne.

WARVI, W. N. \& Gates, O. (1943) Epithelial cysts and cyst tumors of the skin. American Journal of Pathology, 19, 765. 